\title{
ON THE SOLUTIONS OF A MULTI-POINT BVP FOR FRACTIONAL DIFFERENTIAL EQUATIONS WITH IMPULSES
}

\author{
FATMA TOKMAK FEN
}

Received 24 June, 2017

\begin{abstract}
The existence and uniqueness of solutions for a multi-point boundary value problem (BVP) of impulsive fractional differential equations are investigated by means of Schaefer's fixed point theorem and Banach contraction mapping principle. Examples that support the theoretical results are presented.
\end{abstract}

2010 Mathematics Subject Classification: 26A33; 34B37

Keywords: Caputo fractional derivative, impulsive differential equations, fractional differential equations, boundary value problems, Banach contraction mapping principle, Schaefer's fixed point theorem

\section{INTRODUCTION}

Even though it is a 300-year-old topic, fractional calculus has come into prominence in the course of time, and nowadays it has an important role in the modeling of real world phenomena based on fractional derivatives [15]. Models arising from fractional order derivatives are excellent instruments for the description of memory and hereditary properties of various materials and processes [15]. Impulsive differential equations, on the other hand, are capable of describing the dynamics of processes in which abrupt changes occur $[2,17]$. For instance, according to the switching phenomenon, frequency changes or other sudden noises, the states of the electronic devices are often subject to instantaneous perturbations and experience abrupt changes at certain instants $[2,11]$, i.e., they exhibit impulsive effects. Therefore, mathematical models with impulsive effects are more accurate to describe the evolutionary processes of the systems. Fractional order and impulsive differential equations have many applications in various fields such as mechanics, electronics, biology, economics, chaos theory, and neuroscience [1,3-5, 8, 12-14, 19-21].

Various types of impulsive fractional differential equations were considered in the papers [22-27]. Banach, Krasnoselskii's, and Leray-Schauder's fixed point theorems were utilized to show the existence and uniqueness of solutions for a class of fractional order differential switched systems with coupled nonlocal initial and impulsive 
conditions in [24]. The papers [6,27] were concerned with significant results on formulas for solutions of impulsive fractional differential equations. In [27], a boundary value problem (BVP) of order between 1 and 2 is studied. Fixed point theorems were also used in [6] and [27] to show the existence and uniqueness of solutions. On the other hand, the theory of impulsive fractional differential inclusions was improved in [25] by taking into account the existence of PC-mild solutions of such systems with nonlocal conditions when the linear part is a fractional sectorial operator defined on a separable Banach space. Moreover, existence results based on the Banach and Schauder's fixed point theorems were revealed by Wang and Zhang [26] for fractional impulsive systems with Hadamard derivatives. In the present study, we make use of the Banach and Schaefer's fixed point theorems to deal with the existence and uniqueness of solutions of a BVP of fractional differential equations with impulses. In contrast with [27], in this paper multi-points in the boundary conditions are used and the order of the system is between 2 and 3 .

In [10], Liu et al. considered the following nonlinear fractional impulsive differential equation

$$
\left\{\begin{array}{l}
D_{0^{+}}^{\beta} \phi_{p}\left(D_{0^{+}}^{\alpha} u(t)\right)=f(t, u(t)), t \in J^{\prime}, \\
\Delta u\left(t_{k}\right)=I_{k}\left(u\left(t_{k}\right)\right), \Delta \phi_{p}\left(D_{0^{+}}^{\alpha} u\left(t_{k}\right)\right)=b_{k}, k=1,2, \ldots, m, \\
u(0)=u_{0}, D_{0^{+}}^{\alpha} u(0)=u_{1},
\end{array}\right.
$$

where $\alpha, \beta \in(0,1]$ with $\alpha+\beta>1$. The existence and uniqueness of solutions were obtained by means of the Schauder's fixed point theorem and Banach contraction mapping principle. Additionally, in [6], the authors considered the following fractional impulsive differential equation

$$
\left\{\begin{array}{l}
{ }^{C} D_{t}^{q} u(t)=f(t, u(t)), t \in J^{\prime}:=J \backslash\left\{t_{1}, \ldots, t_{m}\right\}, J=[0,1], \\
u\left(t_{k}^{+}\right)=u\left(t_{k}^{-}\right)+y_{k}, k=1,2, \ldots, m, \\
u(0)=u_{0}, y_{k} \in \mathbb{R},
\end{array}\right.
$$

where ${ }^{C} D_{t}^{q}$ is the Caputo fractional derivative of order $q \in(0,1), u_{0} \in \mathbb{R}$, and $f$ : $J \times \mathbb{R} \rightarrow \mathbb{R}$ is a continuous function. Fixed point methods were utilized to obtain some sufficient conditions for the existence of solutions. Moreover, Rehman and Eloe [16] presented a general method for converting an impulsive fractional differential equation to an equivalent integral equation. Using this method, they investigated the existence of solutions for the following four-point impulsive fractional BVP

$$
\left\{\begin{array}{l}
{ }^{C} D^{\alpha} y(x)=f(x, y), x \in J, \\
\Delta y\left(x_{k}\right)=I_{k}\left(u\left(t_{k}\right)\right), \Delta y^{\prime}\left(x_{k}\right)=\bar{I}_{k}\left(y\left(x_{k}\right)\right), k=1,2, \ldots, m, \\
y(0)=\gamma y(\xi), y(1)=\beta y(\eta),
\end{array}\right.
$$

where $1<\alpha<2, \beta, \gamma, \xi, \eta \in \mathbb{R}, \xi, \eta \in(0,1), J=[0,1]$, and $f: J \times \mathbb{R} \rightarrow \mathbb{R}$ is a continuous function. 
Motivated by the above mentioned studies, in this paper, we consider the following $p$-point impulsive fractional BVP

$$
\left\{\begin{array}{l}
{ }^{C} D_{0^{+}}^{\alpha} u(t)=f(t, u(t)), t \in J^{\prime}, \\
\Delta u\left(t_{k}\right)=I_{k}\left(u\left(t_{k}\right)\right), \Delta u^{\prime}\left(t_{k}\right)=\bar{I}_{k}\left(u\left(t_{k}\right)\right), \\
\Delta u^{\prime \prime}\left(t_{k}\right)=\tilde{I}_{k}\left(u\left(t_{k}\right)\right), k=1,2, \ldots, m, \\
a u(0)+b u^{\prime}(0)=\sum_{i=1}^{p-2} c_{i} u\left(\xi_{i}\right), \\
c u(1)+d u^{\prime}(1)=\sum_{i=1}^{p-2} d_{i} u\left(\xi_{i}\right), u^{\prime \prime}(0)=0,
\end{array}\right.
$$

where ${ }^{C} D_{0^{+}}^{\alpha}$ is the Caputo fractional derivative of order $\alpha \in(2,3), a, b, c$, and $d$ are real constants, the functions $f:[0,1] \times \mathbb{R} \rightarrow \mathbb{R}, I_{k}: \mathbb{R} \rightarrow \mathbb{R}, \bar{I}_{k}: \mathbb{R} \rightarrow \mathbb{R}$, and $\tilde{I}_{k}:$ $\mathbb{R} \rightarrow \mathbb{R}$ are continuous in all their arguments, $0<\xi_{1}<\xi_{2}<\cdots<\xi_{p-2}<1, c_{i}, d_{i} \in$ $\mathbb{R}, i=1, \ldots, p-2$, with $\delta:=a c+a d-b c-\sum_{i=1}^{p-2} d_{i} \xi_{i}\left(a-\sum_{i=1}^{p-2} c_{i}\right)+\sum_{i=1}^{p-2} d_{i}(b-$ $\left.\sum_{i=1}^{p-2} c_{i} \xi_{i}\right)+c \sum_{i=1}^{p-2} c_{i} \xi_{i} \neq 0, J=[0,1], 0=t_{0}<t_{1}<\cdots<t_{k}<\cdots<t_{m}<t_{m+1}=1$, $J^{\prime}=J \backslash\left\{t_{1}, t_{2}, \ldots, t_{m}\right\}, \Delta u\left(t_{k}\right)=u\left(t_{k}^{+}\right)-u\left(t_{k}\right)$, and $u\left(t_{k}^{+}\right)=\lim _{t \rightarrow t_{k}^{+}} u(t)$ for each $k=1,2, \ldots, m$. The impulse actions $\Delta u^{\prime}\left(t_{k}\right)$ and $\Delta u^{\prime \prime}\left(t_{k}\right)$ are defined in a similar way to $\Delta u\left(t_{k}\right)$.

The rest of this paper is organized as follows. In Section 2, we provide some necessary definitions and preliminary lemmas, which are key tools for our main results. In Section 3, we make use of the Schaefer's fixed point theorem and Banach contraction mapping principle to prove the existence and uniqueness of solutions of (1.1). Examples are provided in Section 4 to demonstrate the applications of the main results.

\section{Preliminaries}

The following definitions are needed in the remaining parts of the paper.

Definition 1 ([9]). The Riemann-Liouville fractional integral of order $\alpha$ for a function $g:[0, \infty) \rightarrow \mathbb{R}$ is defined as

$$
I_{0^{+}}^{\alpha} g(t)=\frac{1}{\Gamma(\alpha)} \int_{0}^{t}(t-s)^{\alpha-1} g(s) d s, t>0, \alpha>0,
$$

provided the right side is point-wise defined on $[0, \infty)$, where $\Gamma$ is the gamma function. 
Definition 2 ([9]). The Riemann-Liouville derivative of order $\alpha$ for a function $g:[0, \infty) \rightarrow \mathbb{R}$ can be written as

$$
{ }^{L} D_{0^{+}}^{\alpha} g(t)=\frac{1}{\Gamma(n-\alpha)} \frac{d^{n}}{d t^{n}} \int_{0}^{t} \frac{g(s)}{(t-s)^{\alpha+1-n}} d s, t>0,
$$

where $n=[\alpha]+1$ and $[\alpha]$ is the integer part of $\alpha$.

Definition 3 ([9]). The Caputo fractional derivative of order $\alpha>0$ for a continuous function $g:[0, \infty) \rightarrow \mathbb{R}$ can be written as

$$
{ }^{C} D_{0^{+}}^{\alpha} g(t)={ }^{L} D_{0^{+}}^{\alpha}\left[g(t)-\sum_{i=0}^{n-1} \frac{g^{(i)}(0)}{i !} t^{i}\right], t>0,
$$

where $n=[\alpha]+1$ and $[\alpha]$ is the integer part of $\alpha$.

One can confirm that if $g:[0, \infty) \rightarrow \mathbb{R}$ is an $n$ times continuously differentiable function, then

$$
{ }^{C} D_{0^{+}}^{\alpha} g(t)=\frac{1}{\Gamma(n-\alpha)} \int_{0}^{t} \frac{g^{(n)}(s)}{(t-s)^{\alpha+1-n}} d s=I_{0^{+}}^{n-\alpha} g^{(n)}(t),
$$

for $t>0$ and $n-1<\alpha<n$. Moreover, the Caputo derivative of a constant is equal to zero.

We say that a real valued function $u(t)$ defined on $J$ belongs to the space $P C(J, \mathbb{R})$ if it is left-continuous and continuous except, possibly, at the points $t_{k}, k=1,2, \ldots, m$, where it has discontinuities of the first kind. Besides, for a positive integer $\sigma$, we will denote by $P C^{\sigma}(J, \mathbb{R})$ the set of real valued functions whose derivative of order $i$ belongs to the set $P C(J, \mathbb{R})$ for each $i=0,1, \ldots, \sigma$. It is worth noting that the space $P C(J, \mathbb{R})$ is a Banach space endowed with the norm $\|u\|=\sup _{t \in J}|u(t)|$. In the sequel, we will denote $J_{0}=\left[0, t_{1}\right], J_{1}=\left(t_{1}, t_{2}\right], \ldots, J_{m-1}=\left(t_{m-1}, t_{m}\right]$, and $J_{m}=\left(t_{m}, 1\right]$. Moreover, let $A C^{\sigma}(J, \mathbb{R})$ be the set of functions $u(t)$ such that $u^{(\sigma-1)} \in P C(J, \mathbb{R})$ and the restriction of $u^{(\sigma-1)}$ on $J_{k}, k=0,1, \ldots, m$, is absolutely continuous.

Lemma 1 ([16, Corollary 2.5]). Assume $f: J \times \mathbb{R} \rightarrow \mathbb{R}$ is continuous and $n-1<$ $\alpha<n$. Then $u \in \mathcal{A}^{n}(J, \mathbb{R})$ satisfies

$$
{ }^{C} D_{0^{+}}^{\alpha} u(t)=f(t, u(t)), t \in J,
$$

if and only if $u \in P C^{n-1}(J, \mathbb{R})$ and

$$
\begin{aligned}
u(t)= & \sum_{i=0}^{n-1} \frac{u^{(i)}(0)}{i !} t^{i}+\sum_{i=0}^{n-1}\left(\sum_{0<t_{k}<t} \frac{1}{i !} \Delta u^{(i)}\left(t_{k}\right)\left(t-t_{k}\right)^{i}\right) \\
& +\int_{0}^{t} \frac{(t-s)^{\alpha-1}}{\Gamma(\alpha)} f(s, u(s)) d s
\end{aligned}
$$

for $t \in J$. 
Theorem 1 ([18, Schaefer's Fixed Point Theorem]). Let $\mathcal{B}$ be a Banach space and $T: \mathscr{B} \rightarrow \mathscr{B}$ be a completely continuous operator. If the set

$$
\mathscr{D}(\mathscr{B})=\{u \in \mathscr{B}: u=\lambda T u \text { for some } \lambda \in[0,1]\}
$$

is bounded, then T has at least one fixed point.

Lemma 2 ([7, $P C$-type Ascoli-Arzela Theorem]). Let $\Omega \subset P C(J, \mathbb{R})$. Suppose the following conditions are satisfied:

(i) $\Omega$ is uniformly bounded;

(ii) $\Omega$ is equicontinuous in $J_{k}, k=0,1, \ldots, m$.

Then $\Omega$ is a relatively compact subset of $P C(J, \mathbb{R})$.

\section{MAIN RESULTS}

This section is concerned with the existence as well as uniqueness of solutions of the problem (1.1).

In the following lemma, we provide an equivalent integral equation to problem (1.1).

Lemma 3. A function $u(t)$ is a solution of fractional impulsive $B V P(1.1)$ if and only if it is a solution of the following equation

$$
u(t)=\left\{\begin{array}{l}
\int_{0}^{t} \frac{(t-s)^{\alpha-1}}{\Gamma(\alpha)} f(s, u(s)) d s+\frac{1}{\delta} \sum_{i=1}^{p-2} c_{i} F_{u}\left(\xi_{i}\right) \\
\times\left[\left(c+d-\sum_{i=1}^{p-2} d_{i} \xi_{i}\right)-\left(c-\sum_{i=1}^{p-2} d_{i}\right) t\right] \\
+\frac{1}{\delta}\left(\sum_{i=1}^{p-2} d_{i} F_{u}\left(\xi_{i}\right)-\mathcal{A}_{u}\right) \\
\times\left(-b+\sum_{i=1}^{p-2} c_{i} \xi_{i}+\left(a-\sum_{i=1}^{p-2} c_{i}\right) t\right), t \in J_{0}, \\
F_{u}(t)+\frac{1}{\delta} \sum_{i=1}^{p-2} c_{i} F_{u}\left(\xi_{i}\right)\left[\left(c+d-\sum_{i=1}^{p-2} d_{i} \xi_{i}\right)-\left(c-\sum_{i=1}^{p-2} d_{i}\right) t\right] \\
+\frac{1}{\delta}\left(\sum_{i=1}^{p-2} d_{i} F_{u}\left(\xi_{i}\right)-\mathcal{A}_{u}\right)\left(-b+\sum_{i=1}^{p-2} c_{i} \xi_{i}+\left(a-\sum_{i=1}^{p-2} c_{i}\right) t\right) \\
t \in J_{k}, k=1,2, \ldots, m,
\end{array}\right.
$$


where

$$
\begin{aligned}
F_{u}(t)= & \int_{0}^{t} \frac{(t-s)^{\alpha-1}}{\Gamma(\alpha)} f(s, u(s)) d s+\sum_{0<t_{k}<t} I_{k}\left(u\left(t_{k}\right)\right) \\
& +\sum_{0<t_{k}<t} \bar{I}_{k}\left(u\left(t_{k}\right)\right)\left(t-t_{k}\right)+\sum_{0<t_{k}<t} \tilde{I}_{k}\left(u\left(t_{k}\right)\right) \frac{\left(t-t_{k}\right)^{2}}{2},
\end{aligned}
$$

and

$$
\begin{aligned}
\mathcal{A}_{u}= & \int_{0}^{1}\left(\frac{c(1-s)^{\alpha-1}}{\Gamma(\alpha)}+\frac{d(1-s)^{\alpha-2}}{\Gamma(\alpha-1)}\right) f(s, u(s)) d s \\
& +c \sum_{k=1}^{m} I_{k}\left(u\left(t_{k}\right)\right)+\sum_{k=1}^{m} \bar{I}_{k}\left(u\left(t_{k}\right)\right)\left(c\left(1-t_{k}\right)+d\right) \\
& +\frac{1}{2} \sum_{k=1}^{m} \tilde{I}_{k}\left(u\left(t_{k}\right)\right)\left(c\left(1-t_{k}\right)^{2}+2 d\left(1-t_{k}\right)\right) .
\end{aligned}
$$

Proof. Suppose that $u(t)$ is solution of (1.1). One can confirm by means of Lemma 1 that

$$
\begin{gathered}
u(t)=e_{1}+e_{2} t+e_{3} t^{2}+\sum_{0<t_{k}<t} I_{k}\left(u\left(t_{k}\right)\right)+\sum_{0<t_{k}<t} \bar{I}_{k}\left(u\left(t_{k}\right)\right)\left(t-t_{k}\right) \\
+\sum_{0<t_{k}<t} \tilde{I}_{k}\left(u\left(t_{k}\right)\right) \frac{\left(t-t_{k}\right)^{2}}{2}+\int_{0}^{t} \frac{(t-s)^{\alpha-1}}{\Gamma(\alpha)} f(s, u(s)) d s, \\
u^{\prime}(t)=e_{2}+2 e_{3} t+\sum_{0<t_{k}<t} \bar{I}_{k}\left(u\left(t_{k}\right)\right)+\sum_{0<t_{k}<t} \tilde{I}_{k}\left(u\left(t_{k}\right)\right)\left(t-t_{k}\right) \\
\quad+\int_{0}^{t} \frac{(t-s)^{\alpha-2}}{\Gamma(\alpha-1)} f(s, u(s)) d s,
\end{gathered}
$$

and

$$
u^{\prime \prime}(t)=2 e_{3}+\sum_{0<t_{k}<t} \tilde{I}_{k}\left(u\left(t_{k}\right)\right)+\int_{0}^{t} \frac{(t-s)^{\alpha-3}}{\Gamma(\alpha-2)} f(s, u(s)) d s .
$$

Applying the boundary condition $u^{\prime \prime}(0)=0$ in (3.6), we obtain $e_{3}=0$. Now, using the boundary conditions $a u(0)+b u^{\prime}(0)=\sum_{i=1}^{p-2} c_{i} u\left(\xi_{i}\right)$ and $c u(1)+d u^{\prime}(1)=$ $\sum_{i=1}^{p-2} d_{i} u\left(\xi_{i}\right)$, it can be verified that

$$
\left(a-\sum_{i=1}^{p-2} c_{i}\right) e_{1}+\left(b-\sum_{i=1}^{p-2} c_{i} \xi_{i}\right) e_{2}=\sum_{i=1}^{p-2} c_{i} F_{u}\left(\xi_{i}\right)
$$


and

$$
\left(c-\sum_{i=1}^{p-2} d_{i}\right) e_{1}+\left(c+d-\sum_{i=1}^{p-2} d_{i} \xi_{i}\right) e_{2}=\sum_{i=1}^{p-2} d_{i} F_{u}\left(\xi_{i}\right)-\mathcal{A}_{u} .
$$

Solving the linear system of equations (3.7) and (3.8) for $e_{1}$ and $e_{2}$, we get

$$
\begin{aligned}
& e_{1}=\frac{1}{\delta}\left[\left(c+d-\sum_{i=1}^{p-2} d_{i} \xi_{i}\right) \sum_{i=1}^{p-2} c_{i} F_{u}\left(\xi_{i}\right)+\left(-b+\sum_{i=1}^{p-2} c_{i} \xi_{i}\right)\left(\sum_{i=1}^{p-2} d_{i} F_{u}\left(\xi_{i}\right)-\mathcal{A}_{u}\right)\right], \\
& e_{2}=\frac{1}{\delta}\left[\left(a-\sum_{i=1}^{p-2} c_{i}\right)\left(\sum_{i=1}^{p-2} d_{i} F_{u}\left(\xi_{i}\right)-\mathcal{A}_{u}\right)-\left(c-\sum_{i=1}^{p-2} d_{i}\right) \sum_{i=1}^{p-2} c_{i} F_{u}\left(\xi_{i}\right)\right] .
\end{aligned}
$$

Substituting the values of $e_{1}$ and $e_{2}$ in (3.4), we obtain (3.1). Conversely, if $u(t)$ satisfies (3.1), then it is easy to show that $u$ satisfies (1.1). This completes the proof.

Let us define an operator $T: P C(J, \mathbb{R}) \rightarrow P C(J, \mathbb{R})$ as

$$
\begin{array}{r}
T u(t)=F_{u}(t)+\frac{1}{\delta} \sum_{i=1}^{p-2} c_{i} F_{u}\left(\xi_{i}\right)\left[\left(c+d-\sum_{i=1}^{p-2} d_{i} \xi_{i}\right)-\left(c-\sum_{i=1}^{p-2} d_{i}\right) t\right] \\
+\frac{1}{\delta}\left(\sum_{i=1}^{p-2} d_{i} F_{u}\left(\xi_{i}\right)-\mathcal{A}_{u}\right)\left[-b+\sum_{i=1}^{p-2} c_{i} \xi_{i}+\left(a-\sum_{i=1}^{p-2} c_{i}\right) t\right], t \in J,
\end{array}
$$

where $F_{u}(t)$ and $\mathcal{A}_{u}$ are defined as in (3.2) and (3.3), respectively. Clearly, the fixed points of the operator $T$ are the solutions of the problem (1.1).

For the sake of convenience, we define

$$
\begin{aligned}
& \rho_{1}=\sup _{t \in[0,1]} \frac{\left|\left(c+d-\sum_{i=1}^{p-2} d_{i} \xi_{i}\right)-\left(c-\sum_{i=1}^{p-2} d_{i}\right) t\right|}{|\delta|}, \\
& \rho_{2}=\sup _{t \in[0,1]} \frac{\left|-b+\sum_{i=1}^{p-2} c_{i} \xi_{i}+\left(a-\sum_{i=1}^{p-2} c_{i}\right) t\right|}{|\delta|}, \\
& \kappa_{1}=\frac{\left|a-\sum_{i=1}^{p-2} c_{i}\right|}{\left|\delta-\sum_{i=1}^{p-2} d_{i}\right|}, \quad \kappa_{2}=\frac{|\delta|}{|\delta|}
\end{aligned}
$$

A property of the operator $T$ defined by (3.9) is given in the next lemma.

Lemma 4. The operator $T: P C(J, \mathbb{R}) \rightarrow P C(J, \mathbb{R})$ is completely continuous. 
Proof. The operator $T$ is continuous in view of the continuity of the functions $f, I_{k}, \bar{I}_{k}$ and $\tilde{I}_{k}$. Let $\Omega$ be a bounded subset of $P C(J, \mathbb{R})$. There exist positive constants $M_{i}, i=1,2,3,4$, such that $|f(t, u)| \leq M_{1},\left|I_{k}(u)\right| \leq M_{2},\left|\bar{I}_{k}(u)\right| \leq M_{3}$ and $\left|\tilde{I}_{k}(u)\right| \leq M_{4}$ for all $u \in \Omega$.

Thus, we have for $u \in \Omega$ that

$$
\begin{aligned}
& \left|F_{u}\left(\xi_{i}\right)\right| \leq \int_{0}^{\xi_{i}} \frac{\left(\xi_{i}-s\right)^{\alpha-1}}{\Gamma(\alpha)}|f(s, u(s))| d s+\sum_{0<t_{k}<\xi_{i}}\left|I_{k}\left(u\left(t_{k}\right)\right)\right| \\
& +\sum_{0<t_{k}<\xi_{i}}\left|\bar{I}_{k}\left(u\left(t_{k}\right)\right)\right|\left(\xi_{i}-t_{k}\right)+\sum_{0<t_{k}<\xi_{i}}\left|\tilde{I}_{k}\left(u\left(t_{k}\right)\right)\right| \frac{\left(\xi_{i}-t_{k}\right)^{2}}{2} \\
& \leq \frac{\xi_{i}^{\alpha}}{\Gamma(\alpha+1)} M_{1}+m\left(M_{2}+\xi_{i} M_{3}+\frac{\xi_{i}^{2}}{2} M_{4}\right)
\end{aligned}
$$

and

$$
\begin{aligned}
& \left|\mathcal{A}_{u}\right| \leq \int_{0}^{1}\left(\frac{|c|(1-s)^{\alpha-1}}{\Gamma(\alpha)}+\frac{|d|(1-s)^{\alpha-2}}{\Gamma(\alpha-1)}\right)|f(s, u(s))| d s \\
& +|c| \sum_{k=1}^{m}\left|I_{k}\left(u\left(t_{k}\right)\right)\right|+\sum_{k=1}^{m}\left|\bar{I}_{k}\left(u\left(t_{k}\right)\right)\right|\left|c\left(1-t_{k}\right)+d\right| \\
& +\frac{1}{2} \sum_{k=1}^{m}\left|\tilde{I}_{k}\left(u\left(t_{k}\right)\right)\right|\left|c\left(1-t_{k}\right)^{2}+2 d\left(1-t_{k}\right)\right| \\
& \leq\left(\frac{|c|}{\Gamma(\alpha+1)}+\frac{|d|}{\Gamma(\alpha)}\right) M_{1}+m\left(|c| M_{2}+(|c|+|d|) M_{3}\right) \\
& +\frac{m}{2}(|c|+2|d|) .
\end{aligned}
$$

Therefore, one can confirm for $t \in J$ that

$$
\begin{aligned}
& |T u(t)| \leq \int_{0}^{t} \frac{(t-s)^{\alpha-1}}{\Gamma(\alpha)}|f(s, u(s))| d s+\sum_{0<t_{k}<t}\left|I_{k}\left(u\left(t_{k}\right)\right)\right| \\
& +\sum_{0<t_{k}<t}\left|\bar{I}_{k}\left(u\left(t_{k}\right)\right)\right|\left(t-t_{k}\right)+\sum_{0<t_{k}<t}\left|\tilde{I}_{k}\left(u\left(t_{k}\right)\right)\right| \frac{\left(t-t_{k}\right)^{2}}{2} \\
& +\frac{1}{|\delta|} \sum_{i=1}^{p-2}\left|c_{i}\right|\left|F_{u}\left(\xi_{i}\right)\right|\left|\left(c+d-\sum_{i=1}^{p-2} d_{i} \xi_{i}\right)-\left(c-\sum_{i=1}^{p-2} d_{i}\right) t\right| \\
& +\frac{1}{|\delta|}\left(\sum_{i=1}^{p-2}\left|d_{i}\right|\left|F_{u}\left(\xi_{i}\right)\right|+\left|\mathcal{A}_{u}\right|\right)\left|-b+\sum_{i=1}^{p-2} c_{i} \xi_{i}+\left(a-\sum_{i=1}^{p-2} c_{i}\right) t\right|
\end{aligned}
$$




$$
\begin{aligned}
& \leq \frac{M_{1}}{\Gamma(\alpha+1)}+m\left(M_{2}+M_{3}+\frac{M_{4}}{2}\right) \\
& +\sum_{i=1}^{p-2}\left(\rho_{1}\left|c_{i}\right|+\rho_{2}\left|d_{i}\right|\right)\left[\frac{\xi_{i}^{\alpha}}{\Gamma(\alpha+1)} M_{1}+m\left(M_{2}+\xi_{i} M_{3}+\frac{\xi_{i}^{2}}{2} M_{4}\right)\right] \\
& +\rho_{2} M_{1}\left(\frac{|c|}{\Gamma(\alpha+1)}+\frac{|d|}{\Gamma(\alpha)}\right) \\
& +\rho_{2} m\left(|c| M_{2}+(|c|+|d|) M_{3}+\frac{|c|+2|d|}{2} M_{4}\right) .
\end{aligned}
$$

Hence, the operator $T$ is uniformly bounded. On the other hand, for any $t \in J_{k}$, $0 \leq k \leq m$, one can confirm that

$$
\begin{aligned}
& \left|(T u)^{\prime}(t)\right| \leq \int_{0}^{t} \frac{(t-s)^{\alpha-2}}{\Gamma(\alpha-1)}|f(s, u(s))| d s+\sum_{0<t_{k}<t}\left|\bar{I}_{k}\left(u\left(t_{k}\right)\right)\right| \\
& +\sum_{0<t_{k}<t}\left|\tilde{I}_{k}\left(u\left(t_{k}\right)\right)\right|\left(t-t_{k}\right)+\frac{1}{|\delta|} \sum_{i=1}^{p-2}\left|c_{i}\right|\left|F_{u}\left(\xi_{i}\right)\right|\left|c-\sum_{i=1}^{p-2} d_{i}\right| \\
& +\frac{1}{|\delta|}\left(\sum_{i=1}^{p-2}\left|d_{i}\right|\left|F_{u}\left(\xi_{i}\right)\right|+\left|\mathcal{A}_{u}\right|\right)\left|a-\sum_{i=1}^{p-2} c_{i}\right| .
\end{aligned}
$$

Therefore, we have

$$
\begin{aligned}
& \left|(T u)^{\prime}(t)\right| \leq \frac{M_{1}}{\Gamma(\alpha)}+m\left(M_{3}+M_{4}\right) \\
& +\sum_{i=1}^{p-2}\left(\kappa_{1}\left|c_{i}\right|+\kappa_{2}\left|d_{i}\right|\right)\left[\frac{\xi_{i}^{\alpha}}{\Gamma(\alpha+1)} M_{1}+m\left(M_{2}+\xi_{i} M_{3}+\frac{\xi_{i}^{2}}{2} M_{4}\right)\right] \\
& +\kappa_{2} M_{1}\left(\frac{|c|}{\Gamma(\alpha+1)}+\frac{|d|}{\Gamma(\alpha)}\right) \\
& +\kappa_{2} m\left(|c| M_{2}+(|c|+|d|) M_{3}+\frac{|c|+2|d|}{2} M_{4}\right) .
\end{aligned}
$$

The last inequality implies the equicontinuity of $T(\Omega)$ on $J_{k}, k=0,1, \ldots, m$. In accordance with the PC-type Arzela-Ascoli theorem $T(\Omega)$ is relatively compact in $P C(J, \mathbb{R})$. Consequently, the operator $T: P C(J, \mathbb{R}) \rightarrow P C(J, \mathbb{R})$ is completely continuous.

The following conditions are required for the next theorem, which is concerned with the existence of solutions of (1.1).

(C1) There exist nonnegative constants $h$ and $l$ such that

$$
|f(t, u)| \leq h+l|u|, t \in J, u \in \mathbb{R}
$$


(C2) There exist nonnegative constants $h_{k}, l_{k}, \bar{h}_{k}, \bar{l}_{k}, \tilde{h}_{k}, \tilde{l}_{k}$ such that for $u \in \mathbb{R}$, $k=1,2, \ldots, m$,

$$
\left|I_{k}(u)\right| \leq h_{k}+l_{k}|u|,\left|\bar{I}_{k}(u)\right| \leq \bar{h}_{k}+\bar{l}_{k}|u|,\left|\tilde{I}_{k}(u)\right| \leq \tilde{h}_{k}+\tilde{l}_{k}|u| .
$$

Let us define

$$
\begin{aligned}
& K^{*}=\left(\frac{1+\rho_{2}|c|}{\Gamma(\alpha+1)}+\frac{\rho_{2}|d|}{\Gamma(\alpha)}+\sum_{i=1}^{p-2}\left(\rho_{1}\left|c_{i}\right|+\rho_{2}\left|d_{i}\right|\right) \frac{\xi_{i}^{\alpha}}{\Gamma(\alpha+1)}\right) l \\
& +\sum_{i=1}^{p-2}\left(\rho_{1}\left|c_{i}\right|+\rho_{2}\left|d_{i}\right|\right) \sum_{0<t_{k}<\xi_{i}}\left(l_{k}+\xi_{i} \bar{l}_{k}+\frac{\xi_{i}^{2}}{2} \tilde{l}_{k}\right) \\
& +\sum_{k=1}^{m}\left(\left(1+|c| \rho_{2}\right) l_{k}+\left(1+\rho_{2}(|c|+|d|)\right) \bar{l}_{k}+\frac{1+\rho_{2}(|c|+2|d|)}{2} \tilde{l}_{k}\right) .
\end{aligned}
$$

The number $K^{*}$ defined by (3.10) is used in the following theorem.

Theorem 2. Assume that the conditions $(C 1)$ and $(C 2)$ are valid. If $K^{*}<1$, then the problem (1.1) has at least one solution.

Proof. First of all, we know by Lemma 4 that the operator $T: P C(J, \mathbb{R}) \rightarrow$ $P C(J, \mathbb{R})$ defined by equation (3.9) is completely continuous. For $0 \leq \mu \leq 1$, consider the equation

$$
u=\mu T u
$$

If $u$ is a solution of (3.11), then we have for $t \in J$ that

$$
\begin{array}{r}
\left|F_{u}\left(\xi_{i}\right)\right| \leq \int_{0}^{\xi_{i}} \frac{\left(\xi_{i}-s\right)^{\alpha-1}}{\Gamma(\alpha)}(h+l|u(s)|) d s+\sum_{0<t_{k}<\xi_{i}} h_{k}+l_{k}\left|u\left(t_{k}\right)\right| \\
+\sum_{0<t_{k}<\xi_{i}}\left(\bar{h}_{k}+\bar{l}_{k}\left|u\left(t_{k}\right)\right|\right)\left(\xi_{i}-t_{k}\right)+\sum_{0<t_{k}<\xi_{i}}\left(\tilde{h}_{k}+\tilde{l}_{k}\left|u\left(t_{k}\right)\right|\right) \frac{\left(\xi_{i}-t_{k}\right)^{2}}{2} \\
\leq \frac{\xi_{i}^{\alpha} h}{\Gamma(\alpha+1)}+\sum_{0<t_{k}<\xi_{i}}\left(h_{k}+\xi_{i} \bar{h}_{k}+\frac{\xi_{i}^{2}}{2} \tilde{h}_{k}\right)+\frac{\xi_{i}^{\alpha} l}{\Gamma(\alpha+1)}\|u\| \\
+\sum_{0<t_{k}<\xi_{i}}\left(l_{k}+\xi_{i} \bar{l}_{k}+\frac{\xi_{i}^{2}}{2} \tilde{l}_{k}\right)\|u\|
\end{array}
$$


and

$$
\begin{aligned}
& \left|\mathcal{A}_{u}\right| \leq \int_{0}^{1}\left(\frac{|c|(1-s)^{\alpha-1}}{\Gamma(\alpha)}+\frac{|d|(1-s)^{\alpha-2}}{\Gamma(\alpha-1)}\right)|f(s, u(s))| d s \\
& +|c| \sum_{k=1}^{m}\left|I_{k}\left(u\left(t_{k}\right)\right)\right|+\sum_{k=1}^{m}\left|\bar{I}_{k}\left(u\left(t_{k}\right)\right)\right|\left|c\left(1-t_{k}\right)+d\right| \\
& +\frac{1}{2} \sum_{k=1}^{m}\left|\tilde{I}_{k}\left(u\left(t_{k}\right)\right)\right|\left|c\left(1-t_{k}\right)^{2}+2 d\left(1-t_{k}\right)\right| \\
& \leq\left(\frac{|c|}{\Gamma(\alpha+1)}+\frac{|d|}{\Gamma(\alpha)}\right)(h+l\|u\|) \\
& +\sum_{k=1}^{m}\left(|c| h_{k}+(|c|+|d|) \bar{h}_{k}+\frac{1}{2}(|c|+2|d|) \tilde{h}_{k}\right) \\
& +\sum_{k=1}^{m}\left(|c| l_{k}+(|c|+|d|) \bar{l}_{k}+\frac{1}{2}(|c|+2|d|) \tilde{l}_{k}\right)\|u\| .
\end{aligned}
$$

Therefore, we have

$$
\begin{aligned}
& |u(t)|=\mu|T u(t)| \\
& \leq \int_{0}^{t} \frac{(t-s)^{\alpha-1}}{\Gamma(\alpha)}(h+l|u(s)|) d s+\sum_{0<t_{k}<t}\left(h_{k}+l_{k}\left|u\left(t_{k}\right)\right|\right) \\
& +\sum_{0<t_{k}<t}\left(\bar{h}_{k}+\bar{l}_{k}\left|u\left(t_{k}\right)\right|\right)\left(t-t_{k}\right)+\sum_{0<t_{k}<t}\left(\tilde{h}_{k}+\tilde{l}_{k}\left|u\left(t_{k}\right)\right|\right) \frac{\left(t-t_{k}\right)^{2}}{2} \\
& +\rho_{1} \sum_{i=1}^{p-2}\left|c_{i}\right|\left|F_{u}\left(\xi_{i}\right)\right|+\rho_{2} \sum_{i=1}^{p-2}\left|d_{i}\right|\left|F_{u}\left(\xi_{i}\right)\right|+\rho_{2}\left|\mathcal{A}_{u}\right|
\end{aligned}
$$


and thus,

$$
\begin{aligned}
& |u(t)| \leq\left(\frac{1+\rho_{2}|c|}{\Gamma(\alpha+1)}+\frac{\rho_{2}|d|}{\Gamma(\alpha)}+\sum_{i=1}^{p-2}\left(\rho_{1}\left|c_{i}\right|+\rho_{2}\left|d_{i}\right|\right) \frac{\xi_{i}^{\alpha}}{\Gamma(\alpha+1)}\right) h \\
& +\sum_{i=1}^{p-2}\left(\rho_{1}\left|c_{i}\right|+\rho_{2}\left|d_{i}\right|\right) \sum_{0<t_{k}<\xi_{i}}\left(h_{k}+\xi_{i} \bar{h}_{k}+\frac{\xi_{i}^{2}}{2} \tilde{h}_{k}\right) \\
& +\sum_{k=1}^{m}\left(\left(1+\rho_{2}|c|\right) h_{k}+\left(1+\rho_{2}(|c|+|d|)\right) \bar{h}_{k}+\frac{1+\rho_{2}(|c|+2|d|)}{2} \tilde{h}_{k}\right) \\
& +\left[\left(\frac{1+\rho_{2}|c|}{\Gamma(\alpha+1)}+\frac{\rho_{2}|d|}{\Gamma(\alpha)}+\sum_{i=1}^{p-2}\left(\rho_{1}\left|c_{i}\right|+\rho_{2}\left|d_{i}\right|\right) \frac{\xi_{i}^{\alpha}}{\Gamma(\alpha+1)}\right) l\right. \\
& +\sum_{k=1}^{m}\left(\left(1+\rho_{2}|c|\right) l_{k}+\left(1+\rho_{2}(|c|+|d|)\right) \bar{l}_{k}+\frac{1+\rho_{2}(|c|+2|d|)}{2} \tilde{l}_{k}\right) \\
& \left.+\sum_{i=1}^{p-2}\left(\rho_{1}\left|c_{i}\right|+\rho_{2}\left|d_{i}\right|\right) \sum_{0<t_{k}<\xi_{i}}\left(l_{k}+\xi_{i} \bar{l}_{k}+\frac{\xi_{i}^{2}}{2} \tilde{l}_{k}\right)\right]\|u\| .
\end{aligned}
$$

Hence, we have for $t \in J$ that

$$
|u(t)| \leq K+K^{*}\|u\|,
$$

where

$$
\begin{aligned}
& K=\left(\frac{1+\rho_{2}|c|}{\Gamma(\alpha+1)}+\frac{\rho_{2}|d|}{\Gamma(\alpha)}+\sum_{i=1}^{p-2}\left(\rho_{1}\left|c_{i}\right|+\rho_{2}\left|d_{i}\right|\right) \frac{\xi_{i}^{\alpha}}{\Gamma(\alpha+1)}\right) h \\
& +\sum_{i=1}^{p-2}\left(\rho_{1}\left|c_{i}\right|+\rho_{2}\left|d_{i}\right|\right) \sum_{0<t_{k}<\xi_{i}}\left(h_{k}+\xi_{i} \bar{h}_{k}+\frac{\xi_{i}^{2}}{2} \tilde{h}_{k}\right) \\
& +\sum_{k=1}^{m}\left(\left(1+\rho_{2}|c|\right) h_{k}+\left(1+\rho_{2}(|c|+|d|)\right) \bar{h}_{k}+\frac{1+\rho_{2}(|c|+2|d|)}{2} \tilde{h}_{k}\right) .
\end{aligned}
$$

Because $K^{*}<1$, the last inequality implies that

$$
\|u\| \leq \frac{K}{1-K^{*}} .
$$

Therefore, any solution of (3.11) is bounded regardless of the value of $\mu$. According to Theorem 1, the operator $T$ has a fixed point. Consequently, the fractional impulsive multi-point BVP (1.1) has at least one solution.

Now, we will use the Banach contraction mapping principle to prove the existence and uniqueness of solutions for problem (1.1).

The following conditions are required. 
(C3) There exists a positive number $L$ such that $|f(t, u)-f(t, v)| \leq L|u-v|$ for all $t \in J$ and $u, v \in \mathbb{R}$

(C4) There exist positive numbers $L_{1}, L_{2}$, and $L_{3}$ such that $\left|I_{k}(u)-I_{k}(v)\right| \leq$ $L_{1}|u-v|,\left|\bar{I}_{k}(u)-\bar{I}_{k}(v)\right| \leq L_{2}|u-v|$, and $\left|\tilde{I}_{k}(u)-\tilde{I}_{k}(v)\right| \leq L_{3}|u-v|$ for all $u, v \in \mathbb{R}$, and $k=1,2, \ldots, m$.

Theorem 3. Suppose that the conditions (C3), (C4) hold, and

$$
\begin{aligned}
& L<\frac{1}{4}\left(\frac{1+\rho_{2}|c|+\sum_{i=1}^{p-2}\left(\rho_{1}\left|c_{i}\right|+\rho_{2}\left|d_{i}\right|\right) \xi_{i}^{\alpha}}{\Gamma(\alpha+1)}+\frac{\rho_{2}|d|}{\Gamma(\alpha)}\right)^{-1}, \\
& L_{1}<\frac{1}{4 m}\left(\sum_{i=1}^{p-2}\left(\rho_{1}\left|c_{i}\right|+\rho_{2}\left|d_{i}\right|\right)+1+\rho_{2}|c|\right)^{-1}, \\
& L_{2}<\frac{1}{4 m}\left(\sum_{i=1}^{p-2}\left(\rho_{1}\left|c_{i}\right|+\rho_{2}\left|d_{i}\right|\right) \xi_{i}+1+\rho_{2}(|c|+|d|)\right)^{-1}, \\
& L_{3}<\frac{1}{2 m}\left(\sum_{i=1}^{p-2}\left(\rho_{1}\left|c_{i}\right|+\rho_{2}\left|d_{i}\right|\right) \xi_{i}^{2}+1+\rho_{2}(|c|+2|d|)\right)^{-1} .
\end{aligned}
$$

Then, the problem (1.1) has a unique solution.

Proof. Let $u, v \in P C(J, \mathbb{R})$. For each $t \in J$, we have

$$
\begin{aligned}
& |T u(t)-T v(t)| \leq\left|F_{u}(t)-F_{v}(t)\right| \\
& +\frac{1}{|\delta|} \sum_{i=1}^{p-2}\left|c_{i}\right|\left|F_{u}\left(\xi_{i}\right)-F_{v}\left(\xi_{i}\right)\right|\left(c+d-\sum_{i=1}^{p-2} d_{i} \xi_{i}\right)-\left(c-\sum_{i=1}^{p-2} d_{i}\right) t \mid \\
& +\frac{1}{|\delta|}\left(\sum_{i=1}^{p-2}\left|d_{i}\right|\left|F_{u}\left(\xi_{i}\right)-F_{v}\left(\xi_{i}\right)\right|+\left|\mathcal{A}_{u}-\mathcal{A}_{v}\right|\right) \\
& \times\left|-b+\sum_{i=1}^{p-2} c_{i} \xi_{i}+\left(a-\sum_{i=1}^{p-2} c_{i}\right) t\right| \\
& \leq\left[\left(\frac{1+\rho_{2}|c|+\sum_{i=1}^{p-2}\left(\rho_{1}\left|c_{i}\right|+\rho_{2}\left|d_{i}\right|\right) \xi_{i}^{\alpha}}{\Gamma(\alpha+1)}+\frac{\rho_{2}|d|}{\Gamma(\alpha)}\right) L\right. \\
& +\left(\sum_{i=1}^{p-2}\left(\rho_{1}\left|c_{i}\right|+\rho_{2}\left|d_{i}\right|\right)+1+\rho_{2}|c|\right) m L_{1} \\
& +\left(\sum_{i=1}^{p-2}\left(\rho_{1}\left|c_{i}\right|+\rho_{2}\left|d_{i}\right|\right) \xi_{i}+1+\rho_{2}(|c|+|d|)\right) m L_{2} \\
& \left.+\left(\sum_{i=1}^{p-2}\left(\rho_{1}\left|c_{i}\right|+\rho_{2}\left|d_{i}\right|\right) \xi_{i}^{2}+1+\rho_{2}(|c|+2|d|)\right) \frac{m L_{3}}{2}\right]\|u-v\| .
\end{aligned}
$$


Since the inequality

$$
\begin{aligned}
& \left(\frac{1+\rho_{2}|c|+\sum_{i=1}^{p-2}\left(\rho_{1}\left|c_{i}\right|+\rho_{2}\left|d_{i}\right|\right) \xi_{i}^{\alpha}}{\Gamma(\alpha+1)}+\frac{\rho_{2}|d|}{\Gamma(\alpha)}\right) L \\
& +\left(\sum_{i=1}^{p-2}\left(\rho_{1}\left|c_{i}\right|+\rho_{2}\left|d_{i}\right|\right)+1+\rho_{2}|c|\right) m L_{1} \\
& +\left(\sum_{i=1}^{p-2}\left(\rho_{1}\left|c_{i}\right|+\rho_{2}\left|d_{i}\right|\right) \xi_{i}+1+\rho_{2}(|c|+|d|)\right) m L_{2} \\
& +\left(\sum_{i=1}^{p-2}\left(\rho_{1}\left|c_{i}\right|+\rho_{2}\left|d_{i}\right|\right) \xi_{i}^{2}+1+\rho_{2}(|c|+2|d|)\right) \frac{m L_{3}}{2} \\
& <1
\end{aligned}
$$

holds, $T$ is a contraction mapping. According to the Banach contraction mapping principle, $T$ has a unique fixed point in $P C(J, \mathbb{R})$, which is a solution of the problem (1.1).

Examples that support the theoretical results are presented in the next section.

\section{EXAMPLES}

\section{Example 1}

Let us take into account the fractional order impulsive BVP

$$
\left\{\begin{array}{l}
{ }^{C} D_{0^{+}}^{\frac{9}{4}} u(t)=\frac{1}{2} \cos (\sqrt{t})+\frac{3}{20} \tanh (u(t)), t \in[0,1] \backslash\left\{t_{1}, t_{2}\right\}, \\
\Delta u\left(t_{k}\right)=\frac{(-1)^{k}}{5}+\frac{1}{40} u\left(t_{k}\right), \Delta u^{\prime}\left(t_{k}\right)=\frac{1}{10} \sin \left(u\left(t_{k}\right)\right), \\
\Delta u^{\prime \prime}\left(t_{k}\right)=\frac{1}{30} \arctan \left(u\left(t_{k}\right)\right), k=1,2 \\
-3 u(0)+2 u^{\prime}(0)=-8 u\left(\frac{1}{4}\right)+4 u\left(\frac{3}{4}\right), \\
u(1)-2 u^{\prime}(1)=7 u\left(\frac{1}{4}\right)-u\left(\frac{3}{4}\right), u^{\prime \prime}(0)=0,
\end{array}\right.
$$

where $t_{1}=\frac{3}{10}$ and $t_{2}=\frac{4}{5}$.

$\operatorname{BVP}(4.1)$ is in the form of (1.1) with $\alpha=\frac{9}{4}, m=2, p=4, a=-3, b=2, c=1$, $d=-2 c_{1}=-8, c_{2}=4, d_{1}=7, d_{2}=-1, \xi_{1}=\frac{1}{4}, \xi_{2}=\frac{3}{4}, f(t, u)=\frac{1}{2} \cos (\sqrt{t})+$ $\frac{3}{20} \tanh (u), I_{k}(u)=\frac{(-1)^{k}}{5}+\frac{1}{40} u, \bar{I}_{k}(u)=\frac{1}{10} \sin (u)$, and $\tilde{I}_{k}(u)=\frac{1}{30} \arctan (u)$. It can be verified that $\delta=7, \rho_{1}=\frac{3}{7}$, and $\rho_{2}=\frac{1}{7}$ for (4.1). Moreover, conditions $(C 1)$ 
and (C2) are satisfied with $h=\frac{1}{2}, l=\frac{3}{20}, h_{1}=h_{2}=\frac{1}{5}, l_{1}=l_{2}=\frac{1}{40}, \bar{h}_{1}=\bar{h}_{2}=$ $\tilde{h}_{1}=\tilde{h}_{2}=0, \bar{l}_{1}=\bar{l}_{2}=\frac{1}{10}, \tilde{l}_{1}=\tilde{l}_{2}=\frac{1}{30}$. Therefore, according to Theorem 2, BVP (4.1) has at least one solution.

\section{Example 2}

Consider the fractional order impulsive BVP

$$
\left\{\begin{array}{l}
{ }^{C} D_{0^{+}}^{\frac{5}{2}} u(t)=\sin t+\frac{7}{200} \arctan (u(t)), t \in[0,1] \backslash\left\{\frac{3}{4}\right\}, \\
\Delta u\left(\frac{3}{4}\right)=\frac{e^{u\left(\frac{3}{4}\right)}}{50\left(1+e^{u\left(\frac{3}{4}\right)}\right)}, \Delta u^{\prime}\left(\frac{3}{4}\right)=\frac{1}{100} u\left(\frac{3}{4}\right), \\
\Delta u^{\prime \prime}\left(\frac{3}{4}\right)=\frac{1}{20} \cos u\left(\frac{3}{4}\right), \\
u(0)+\frac{1}{2} u^{\prime}(0)=-u\left(\frac{1}{2}\right)+8 u\left(\frac{7}{8}\right), \\
-u(1)+u^{\prime}(1)=4 u\left(\frac{1}{2}\right)-8 u\left(\frac{7}{8}\right), u^{\prime \prime}(0)=0 .
\end{array}\right.
$$

One can confirm that (4.2) is in the form of (1.1) with $\alpha=\frac{5}{2}, m=1, t_{1}=\frac{3}{4}$, $p=4, a=d=1, b=\frac{1}{2}, c=-1=c_{1}, c_{2}=8, d_{1}=4, d_{2}=-8, \xi_{1}=\frac{1}{2}, \xi_{2}=\frac{7}{8}$, $f(t, u)=\sin t+\frac{7}{200} \arctan (u), I_{k}(u)=\frac{e^{u}}{50\left(1+e^{u}\right)}, \bar{I}_{k}(u)=\frac{1}{100} u$, and $\tilde{I}_{k}(u)=$ $\frac{1}{20} \cos u$. One can confirm that $\delta=-12, \rho_{1}=\frac{5}{12}$, and $\rho_{2}=\frac{1}{2}$ for (4.2). Conditions of Theorem 3 are valid for the problem with $L=0.035, L_{1}=0.02, L_{2}=0.01$ and $L_{3}=0.05$. Thus, the fractional impulsive BVP (4.2) has a unique solution.

\section{REFERENCES}

[1] E. Ahmed, A. M. A. El-Sayed, and H. A. A. El-Saka, "Equilibrium points, stability and numerical solutions of fractional-order predator-prey and rabies models," J. Math. Anal. Appl., vol. 325, no. 1, pp. 542-553, 2007, doi: 10.1016/j.jmaa.2006.01.087.

[2] M. Akhmet, Principles of Discontinuous Dynamical Systems. Springer, New York, 2010. doi: 10.1007/978-1-4419-6581-3.

[3] M. Akhmet and M. O. Fen, Replication of Chaos in Neural Networks, Economics and Physics. Higher Education Press, Beijing; Springer, Heidelberg, 2016. doi: 10.1007/978-3-662-47500-3.

[4] M. O. Fen and M. Akhmet, "Impulsive SICNNs with chaotic postsynaptic currents," Discrete Cont. Dyn.-B, vol. 21, no. 4, pp. 1119-1148, 2016, doi: 10.3934/dcdsb.2016.21.1119.

[5] M. O. Fen and F. Tokmak Fen, "Homoclinic and heteroclinic motions in hybrid systems with impacts," Math. Slovaca, vol. 67, no. 5, pp. 1179-1188, 2017, doi: 10.1515/ms-2017-0041. 
[6] M. Fečkan, Y. Zhou, and J. Wang, "On the concept and existence of solution for impulsive fractional differential equations," Commun. Nonlinear Sci. Numer. Simul., vol. 17, no. 7, pp. 30503060, 2012, doi: 10.1016/j.cnsns.2011.11.017.

[7] D. Guo, J. Sun, and Z. Liu, Functional Method for Nonlinear Ordinary Differential Equation. Shandong Science and Technolog Press, Jinan, 1995.

[8] R. Hilfer, Applications of Fractional Calculus in Physics. World Scientific, Hackensack, NJ, 2001.

[9] A. A. Kilbas, H. M. Srivastava, and J. J. Trujillo, Theory and Applications of Fractional Differential Equations. Elsevier Science B.V., Amsterdam, 2006, vol. 204.

[10] Z. Liu, L. Lu, and I. Szántó, "Existence of solutions for fractional impulsive differential equations with $p$-Laplacian operator," Acta Math. Hungar., vol. 141, no. 3, pp. 203-219, 2013, doi: 10.1007/s10474-013-0305-0.

[11] J. Lu, D. Ho, J. Cao, and J. Kurths, "Exponential synchronization of linearly coupled neural networks with impulsive disturbances," IEEE Trans. Neural Netw., vol. 22, no. 2, pp. 329-335, 2011, doi: 10.1109/TNN.2010.2101081.

[12] B. Lundstrom, M. Higgs, W. Spain, and A. Fairhall, "Fractional differentiation by neocortical pyramidal neurons," Nat. Neurosci., vol. 11, pp. 1335-1342, 2008, doi: 10.1038/nn.2212.

[13] J. A. T. Machado and M. E. Mata, "A fractional perspective to the bond graph modelling of world economies," Nonlinear Dynam., vol. 80, no. 4, pp. 1839-1852, 2015, doi: 10.1007/s11071-0141334-0.

[14] F. Mainardi, Fractional Calculus and Waves in Linear Viscoelasticity. Imperial College Press, London, 2010. doi: 10.1142/9781848163300.

[15] I. Podlubny, Fractional Differential Equations. Academic Press, Inc., San Diego, CA, 1999, vol. 198.

[16] M. u. Rehman and P. W. Eloe, "Existence and uniqueness of solutions for impulsive fractional differential equations," Appl. Math. Comput., vol. 224, pp. 422-431, 2013, doi: 10.1016/j.amc.2013.08.088.

[17] A. M. Samoilenko and N. A. Perestyuk, Impulsive Differential Equations. World Scientific Publishing Co., Inc., River Edge, NJ, 1995, vol. 14, doi: 10.1142/9789812798664.

[18] D. R. Smart, Fixed point theorems. Cambridge University Press, London-New York, 1974.

[19] V. E. Tarasov, "Fractional vector calculus and fractional Maxwell's equations," Ann. Physics, vol. 323, no. 11, pp. 2756-2778, 2008, doi: 10.1016/j.aop.2008.04.005.

[20] F. Tokmak Fen and I. Yaslan Karaca, "Existence of positive solutions for nonlinear second-order impulsive boundary value problems on time scales," Mediterr. J. Math., vol. 13, no. 1, pp. 191204, 2016, doi: 10.1007/s00009-014-0494-y.

[21] V. V. Uchaikin, Fractional Derivatives for Physicists and Engineers. Volume I. Higher Education Press, Beijing; Springer, Heidelberg, 2013. doi: 10.1007/978-3-642-33911-0.

[22] J. Wang, M. Fečkan, and Y. Zhou, "Nonlocal impulsive fractional differential inclusions with fractional sectorial operators on Banach spaces," Dynamics of PDE, vol. 8, no. 4, pp. 345-361, 2011.

[23] J. Wang, M. Fečkan, and Y. Zhou, "A survey on impulsive fractional differential equations," Fract. Calc. Appl. Anal., vol. 19, no. 4, pp. 806-831, 2016, doi: 10.1515/fca-2016-0044.

[24] J. Wang, M. Fečkan, and Y. Zhou, "Fractional order differential switched systems with coupled nonlocal initial and impulsive conditions," Bull. Sci. Math., vol. 141, no. 7, pp. 727-746, 2017, doi: 10.1016/j.bulsci.2017.07.007.

[25] J. Wang, A. G. Ibrahim, and M. Fečkan, "On the concept and existence of solution for impulsive fractional differential equations," Appl. Math. Comput., vol. 257, pp. 103-118, 2015, doi: 10.1016/j.amc.2014.04.093. 
[26] J. Wang and Y. Zhang, "On the concept and existence of solutions for fractional impulsive systems with Hadamard derivatives," Appl. Math. Lett., vol. 39, pp. 85-90, 2015, doi: 10.1016/j.aml.2014.08.015

[27] J. Wang, Y. Zhou, and M. Fečkan, "On recent developments in the theory of boundary value problems for impulsive fractional differential equations," Comput. Math. Appl., vol. 64, no. 10, pp. 3008-3020, 2012, doi: 10.1016/j.camwa.2011.12.064.

\section{Author's address}

\section{Fatma Tokmak Fen}

Department of Mathematics, Gazi University, 06500 Teknikokullar, Ankara, Turkey

E-mail address: fatma.tokmakk@gmail.com 